\title{
The quest for purely virtual quanta: fakeons versus Feynman-Wheeler particles
}

\author{
Damiano Anselmi \\ Dipartimento di Fisica "Enrico Fermi", Università di Pisa and INFN, Sezione di Pisa, \\ Largo B. Pontecorvo 3, Pisa 56127, Italy \\ E-mail: damiano.anselmi@unipi.it
}

ABSTRACT: The search for purely virtual quanta has attracted interest in the past. We consider various proposals and compare them to the concept of fake particle, or "fakeon". In particular, the Feynman-Wheeler propagator, which amounts to using the Cauchy principal value inside Feynman diagrams, violates renormalizability, unitarity and stability, due to the coexistence of the prescriptions $\pm i \epsilon$. We contrast the Feynman, fakeon and FeynmanWheeler prescriptions in ordinary as well as cut diagrams. The fakeon does not have the problems of the Feynman-Wheeler propagator and emerges as the correct concept of purely virtual quantum. It allows us to make sense of quantum gravity at the fundamental level, and places it on an equal footing with the standard model. The resulting theory of quantum gravity is perturbative up to an incredibly high energy.

KEywords: Beyond Standard Model, Models of Quantum Gravity, Renormalization Regularization and Renormalons

ARXIV EPRINT: 2001.01942 


\section{Contents}

1 Introduction 1

2 Bubble diagram with FW particles: nonlocal divergent part 3

3 Bubble diagram with FW particles: finite part 6

4 Unitarity 1

5 Stability 11

$6 \quad$ Fakeons as purely virtual quanta 12

7 Lower dimensions $\quad 15$

8 Fakeons, God's energy and the infinite desert $\quad 15$

9 Conclusions 17

\section{Introduction}

A normal particle can be real or virtual, depending on whether it is on shell or off shell. For a variety of reasons, it is interesting to consider the possibility of having quanta that are purely virtual. This means quanta that propagate inside the scattering processes, but cannot be directly observed, because they do not belong to the physical spectrum, i.e. the set of asymptotic states.

Sings of interest for purely virtual particles have been present in the literature for a long time, at the classical and quantum levels. Dirac considered the Abraham-Lorentz force in classical electrodynamics, which effectively describes the recoil on an accelerated pointlike electric charge due to the emission of radiation. It is well-known that, under certain assumptions, this effect can be described by means of a higher-derivative equation, which has undesirable runaway solutions. Dirac "virtualized" the runaway solutions by trading them for violations of microcausality $[1,2]$. A similar trick can be applied to higher-derivative gravity [3, 4], still at the classical level. Feynman and Wheeler studied a version of classical electrodynamics where the Green function is half the sum of the retarded and advanced potentials $[5,6]$ and recovered causality by means of an involved emitterabsorber theory. The same Green function, that is to say, the Cauchy principal value of the unprescribed propagator $1 /\left(p^{2}-m^{2}\right)$, appears in many contexts. Differently from the retarded and advanced potentials and the Feynman propagator, it does not contain the on shell $\delta$ function, so it may be viewed as a candidate to describe purely virtual particles. As such, Bollini and Rocca in ref. [7] and later Plastino and Rocca in ref. [8] studied it at the quantum level and claimed they could make some sense out of it.

The interest for purely virtual quanta was not misplaced, but, for a variety of reasons that we explain in this paper, the principal value of $1 /\left(p^{2}-m^{2}\right)$ cannot be the right answer at the quantum level, because it generates serious problems when it is used inside 
Feynman diagrams. First, it violates the locality of counterterms by generating nonlocal ultraviolet divergences similar to those of ref. [9], whose removal destroys the basic structure of the theory. Second, it generates imaginary parts that are inconsistent with the optical theorem and unitarity. Third, it violates stability, since the ones that are normally known as pseudothresholds become true thresholds, which leads to processes where the incoming energy is equal to a difference of frequencies, rather than the sum.

The problems are due to the coexistence of the $\pm i \epsilon$ prescriptions within the same diagram. In ref. [9] Aglietti and the current author showed that for finite $\epsilon$, these types of diagrams have nonlocal divergent parts in Minkowski spacetime. Although the analysis of [9] was performed in higher derivative theories, its main conclusions extend to infinitesimal widths and the principal-value propagator.

To fix a bit of terminology, the principal-value propagator will be called FeynmanWheeler (FW) propagator, when it is used inside Feynman diagrams. The degrees of freedom it propagates will be called FW particles.

In the end, the FW particles lack a consistent physical interpretation and are even problematic at the mathematical level, due to the nonlocal divergences. The correct notion of purely virtual quantum turns out to be the fakeon [10, 11], which is encoded in a new prescription to quantize the poles of a free propagator. Although the fakeon propagator tends to the principal value of $1 /\left(p^{2}-m^{2}\right)$ in the classical free-field limit, it radically differs from it at the quantum level and when self-interactions are turned on [12]. Not surprisingly, the nature of a purely virtual quantum is... purely quantum, so the classical limit is not enough to infer the quantum nature of the fakeon.

The fakeons do not violate stability, because the pseudothresholds play no significant role. Actually, the thresholds associated with a Feynman diagram that involves fakeons are exactly the ones that are found by means of the usual Feynman prescription. The locality of counterterms is still valid, because the divergent part of a diagram coincides with the one of its Euclidean version [11]. Finally, unitarity is fulfilled, since the imaginary parts of the amplitudes do not receive contributions from the thresholds of the processes that involve fakeons. This means that such processes have zero chances to turn the fakeons into real particles, which is the reason why the fakeons remain virtual and do not belong to the set of asymptotic states.

It is worth to stress that the properties just listed hold at the fundamental level, not just at the effective one. In no other case we can really drop a particle from the physical spectrum (letting aside gauge artifacts, such as the Faddeev-Popov ghosts and the unphysical modes of the gauge fields). In particular, we cannot drop unstable particles. For example, although the muon decays, it can be observed before it does. In the end, only the fakeon fulfills the requirements to be called a purely virtual quantum, which is possible because it is a thoroughly new concept.

An important application of the fakeon prescription is that it allows us to make sense of quantum gravity as a quantum field theory [10], by reconciling unitarity and renormalizability. The resulting theory propagates the graviton, a massive scalar field $\phi$, which can be either a physical particle or a fakeon, and a massive spin-2 fakeon $\chi_{\mu \nu}$. The masses $m_{\phi}$ and $m_{\chi}$ are free parameters. Presumably, their values are smaller than the Planck 
mass, or even much smaller. If so, the quantum gravity theory obtained from the fakeon quantization turns out to be perturbative up to an unbelievably high energy, an energy so high that we think it deserves to be named "God's energy". At the same time, it leads to the troubling scenario of an infinite desert with no new physics between the Planck scale and God's energy.

Something that the Dirac virtualization, the FW idea and the fakeon have in common is that they all lead to violations of causality at very small distances (in different ways in the three cases) $[12,16]$. Physically, the violation of microcausality is not a high price to pay, since we do not have arguments in favor of absolute causality. It must also be recalled that it is not straightforward to define the concept of causality in quantum field theory, even in flat space, ${ }^{1}$ since it is hard to accurately locate spacetime points when we describe on-shell particles by means of relativistic wave packets. Both the Bogoliubov condition [18] and the Lehmann-Symanzik-Zimmermann requirement that fields commute at spacelike separated points [19] are off shell and do not easily translate into properties of the $S$ matrix.

Clearly, the ideas of causality and time ordering make sense only as long as the notion of time makes sense. At the experimental level, the shortest time interval we can measure directly is $10^{-17}-10^{-18}$ seconds $[13,14]$. The theory of quantum gravity based on fakeons predicts that time loses meaning at distances smaller than the Compton wavelength $1 / m_{\chi}$ of the fakeon $\chi_{\mu \nu}$ (or the larger between $1 / m_{\chi}$ and $1 / m_{\phi}$, if both $\chi_{\mu \nu}$ and $\phi$ are fakeons). This means $10^{-36} \mathrm{~s}$, i.e. almost twenty orders of magnitude away from our present accuracy, if the masses $m_{\chi}, m_{\phi}$ are assumed to be around $10^{12} \mathrm{GeV}$. The simplest attempts we can think of to amplify the effect do not lead too far. Actually, in some cases the universe conspires to recover causality for free [15]. In the classical limit, we may have to downgrade the violation of causality to an unusual form of the equations of motion and a fuzziness of their solutions $[12,15]$, which still lead to predictions that can be tested experimentally. Finally, we stress that even if time loses sense at small scales, scattering processes in momentum space make sense to arbitrarily high energies.

The paper is organized as follows. In section 2 we calculate the nonlocal divergent parts of the bubble diagrams involving Feynman-Wheeler particles. In section 3 we evaluate their finite parts. In section 4 we analyze their imaginary parts and show that they violate the optical theorem. In section 5 we discuss the problems of the FW particles with stability. In section 6 we compute the bubble diagrams with circulating fakeons and show that the fakeon prescription is consistent with the locality of counterterms, unitarity and stability. In section 7 we extend the analysis to three and two spacetime dimensions, which confirm the properties found in four dimensions. In section 8 we discuss the impact of the fakeon idea on the perturbative nature of quantum gravity. Section 9 contains the conclusions.

\section{Bubble diagram with FW particles: nonlocal divergent part}

In this section we show that the FW propagator

$$
\mathcal{P} \frac{1}{p^{2}-m^{2}}
$$

\footnotetext{
${ }^{1}$ See section 6.1 of ref. [17] for an illuminating discussion.
} 
where $\mathcal{P}$ denotes the Cauchy principal value, leads to nonlocal ultraviolet divergences when it is used inside Feynman diagrams. Note that in this paper we do not attach factors of $i$ to the vertices and the propagators.

Specifically, we consider the bubble diagram where one virtual particle is quantized by means of the Feynman $+i \epsilon$ prescription and the other virtual particle has the propagator (2.1). The loop integral reads

$$
\Sigma_{\mathrm{FW}}(p)=\int_{k_{s} \leqslant \Lambda} \frac{\mathrm{d}^{3} \mathbf{k}}{(2 \pi)^{3}} \int_{-\infty}^{+\infty} \frac{\mathrm{d} k_{0}}{2 \pi} \frac{1}{(p-k)^{2}-m_{1}^{2}+i \epsilon} \mathcal{P} \frac{1}{k^{2}-m_{2}^{2}},
$$

where $k=\left(k^{0}, \mathbf{k}\right), k_{s}=|\mathbf{k}|$ and $\Lambda$ is a cutoff. We take different masses $m_{1} \neq m_{2}$, because the nonlocal divergent part that we want to compute vanishes for $m_{1}=m_{2}$.

We anticipate that, apart from the locality of counterterms, which is lost, other common properties continue to hold. For example, although the cutoff $\Lambda$ breaks Lorentz invariance, the breaking does not affect the finite parts and the logarithmic divergences we find below. In section 3 we switch to the dimensional regularization, which is manifestly Lorentz invariant.

Writing

$$
\mathcal{P} \frac{1}{k^{2}-m_{2}^{2}}=\frac{1}{2}\left(\frac{1}{k^{2}-m_{2}^{2}-i \epsilon}+\frac{1}{k^{2}-m_{2}^{2}+i \epsilon}\right),
$$

we can decompose $\Sigma_{\mathrm{FW}}(p)$ as the sum

$$
\Sigma_{\mathrm{FW}}(p)=\frac{1}{2}\left(\Sigma(p)+\Sigma^{\prime}(p)\right)
$$

where $\Sigma(p)$ is the usual self-energy, with two propagators quantized à la Feynman [its expression being given by formula (6.1) of section 6], and

$$
\Sigma^{\prime}(p)=\int_{k_{s} \leqslant \Lambda} \frac{\mathrm{d}^{3} \mathbf{k}}{(2 \pi)^{3}} \int_{-\infty}^{+\infty} \frac{\mathrm{d} k_{0}}{2 \pi} \frac{1}{(p-k)^{2}-m_{1}^{2}+i \epsilon} \frac{1}{k^{2}-m_{2}^{2}-i \epsilon} .
$$

Since $\Sigma(p)$ does not contain nonlocal divergent parts, we omit it for the moment and focus on $\Sigma^{\prime}(p)$. The crucial point is that $\Sigma^{\prime}(p)$ involves both prescriptions $\pm i \epsilon$. The nonlocal divergent part is due to the conflict between them.

Writing $p=\left(p^{0}, \mathbf{p}\right)$, we apply the residue theorem to evaluate the integral on the loop energy $k_{0}$, which gives

$$
\Sigma^{\prime}(p)=\frac{i}{4} \int_{k_{s} \leqslant \Lambda} \frac{\mathrm{d}^{3} \mathbf{k}}{(2 \pi)^{3}} \frac{1}{\omega_{1} \omega_{2}}\left(\frac{1}{p^{0}-\omega_{1}+\omega_{2}+i \epsilon}-\frac{1}{p^{0}+\omega_{1}-\omega_{2}-i \epsilon}\right),
$$

where $\omega_{1}=\sqrt{k_{s}^{2}+p_{s}^{2}-2 k_{s} p_{s} u+m_{1}^{2}}, \omega_{2}=\sqrt{k_{s}^{2}+m_{2}^{2}}, p_{s}=|\mathbf{p}|$ and $u=\cos \theta, \theta$ being the angle between $\mathbf{k}$ and $\mathbf{p}$.

Let us start from the case $p^{2} \geqslant 0$ and choose a reference frame where $p_{s}=0$. Then, it is easy to check that

$$
\left(\omega_{1}-\omega_{2}\right)^{2} \leqslant\left(m_{1}-m_{2}\right)^{2}
$$


If we assume $p^{2}>\left(m_{1}-m_{2}\right)^{2},(2.4)$ gives $\left(p^{0}\right)^{2}>\left(\omega_{1}-\omega_{2}\right)^{2}$, which means that the $\pm i \epsilon$ prescriptions in (2.3) are unnecessary. Then we obtain

$$
\Sigma^{\prime}(p)=\frac{i}{8 \pi^{2}} \int_{0}^{\Lambda} \frac{k_{s}^{2} \mathrm{~d} k_{s}}{\omega_{1} \omega_{2}}\left(\frac{1}{p^{0}-\omega_{1}+\omega_{2}}-\frac{1}{p^{0}+\omega_{1}-\omega_{2}}\right) .
$$

It is easy to check that the integrand behaves as

$$
\frac{m_{1}^{2}-m_{2}^{2}}{\left(p^{0}\right)^{2} k_{s}}+\mathcal{O}\left(\frac{1}{k_{s}^{3}}\right)
$$

for large $k_{s}$, which means that $\Sigma^{\prime}(p)$ has the nonlocal ultraviolet divergence

$$
\Sigma_{\mathrm{div}}^{\prime}(p)=\frac{i \ln \Lambda^{2}}{(4 \pi)^{2}} \frac{m_{1}^{2}-m_{2}^{2}}{p^{2}}
$$

To check Lorentz invariance, we repeat the calculation for $p^{2}>0$ without taking $p_{s}=0$. In that case, the inequality (2.4) non longer holds, in general. However, we have $\left(p^{0}\right)^{2}-\left(\omega_{1}-\omega_{2}\right)^{2}=p^{2}+p_{s}^{2}\left(1-u^{2}\right)+\mathcal{O}\left(1 / k_{s}\right)$, so when $k_{s}$ is sufficiently large we do have $\left(p^{0}\right)^{2}>\left(\omega_{1}-\omega_{2}\right)^{2}$ and we can repeat the arguments above, ignoring the $\pm i \epsilon$ prescriptions. At the end, we find the same nonlocal divergent part (2.6).

Finally, if $p^{2}<0$ we take $p^{0}=0$. We need to compute

$$
\Sigma^{\prime}(p)=\frac{i}{8 \pi^{2}} \int_{0}^{\Lambda} \frac{k_{s}^{2} \mathrm{~d} k_{s}}{\omega_{1} \omega_{2}} \int_{-1}^{1} \frac{\mathrm{d} u}{\omega_{2}-\omega_{1}+i \epsilon} .
$$

For large $k_{s}$, the divergent part is given by

$$
\begin{aligned}
\Sigma_{\text {div }}^{\prime}(p) & =\frac{i}{8 \pi^{2}} \int_{0}^{\Lambda} \mathrm{d} k_{s} \int_{-1}^{1} \mathrm{~d} u\left[\frac{1}{p_{s}(u+i \epsilon)}+\frac{m_{1}^{2}-m_{2}^{2}+p_{s}^{2}\left(1+u^{2}\right)}{2 k_{s} p_{s}^{2}(u+i \epsilon)^{2}}\right] \\
& =\frac{\Lambda}{8 \pi p_{s}}-\frac{i\left(m_{1}^{2}-m_{2}^{2}\right)}{(4 \pi)^{2} p_{s}^{2}} \ln \Lambda^{2}
\end{aligned}
$$

(after suitably rescaling $\epsilon$ ). The logarithmic divergence agrees with (2.6). In addition, we have a nonlocal linear divergence. We do not attach a particular meaning to it, because it depends on the regulator and indeed disappears using the dimensional regularization (see next section).

The nonlocal divergent parts we have just calculated are very similar to those found in ref. [9] in higher-derivative theories with finite widths $\epsilon$. They have the same origin: the coexistence of propagators with both positive and negative widths within the same Feynman diagram.

Note that the nonlocal divergent part (2.6) cancels out in the bubble diagram

$$
\Sigma_{\mathrm{FW}-\mathrm{FW}}(p)=\mathcal{P} \int \frac{\mathrm{d}^{D} k}{(2 \pi)^{D}} \frac{1}{(p-k)^{2}-m_{1}^{2}} \frac{1}{k^{2}-m_{2}^{2}}=\frac{1}{2}\left(\Sigma_{\mathrm{FW}}(p)+\Sigma_{\mathrm{FW}}^{*}(p)\right)
$$


made of two FW propagators, since the result must be symmetric under the exchange of $m_{1}$ and $m_{2}$. For future purposes, we have written (2.8) in arbitrary dimension $D$.

Since (2.6) does not cancel out in mixed bubble diagrams $\Sigma_{\mathrm{FW}}(p)$, it must be subtracted away by means of a nonlocal counterterm, which destroys the locality of the theory. In the end, we must conclude that a theory propagating FW particles is mathematically unacceptable.

\section{Bubble diagram with FW particles: finite part}

In this section we work out the finite part of $\Sigma^{\prime}(p)$, which allows us to highlight the physical problems of the theories that propagate $\mathrm{FW}$ particles.

We start by taking $m_{2}=0$. For $p^{2}>0$ we choose a reference frame where $p_{s}=0$. Using (2.3) and denoting $m_{1}$ by $m$, we obtain

$$
i(4 \pi)^{2} \Sigma^{\prime}(p)=-\frac{m^{2}}{p^{2}} \ln \frac{4 \Lambda^{2}}{m^{2}}-\frac{p^{2}-m^{2}}{p^{2}} \ln \frac{p^{2}-m^{2}+i \epsilon}{p^{2}} .
$$

Note the negative imaginary part

$$
2 \operatorname{Im}\left[-i \Sigma^{\prime}(p)\right]=-\frac{m^{2}-p^{2}}{8 \pi p^{2}} \theta\left(m^{2}-p^{2}\right),
$$

which is problematic for unitarity (see next section).

To switch to the case of generic masses, it is convenient to use the dimensional regularization [20-22]. The calculation by means of Feynman parameters exhibits some unexpected features. Indeed, we cannot use the Feynman parameters for $\Sigma^{\prime}(p)$ as we would ordinarily do. We must first change the sign of one propagator to have $+i \epsilon$ in both of them. After a translation of the loop momentum $k$, we get

$$
\begin{aligned}
\Sigma^{\prime}(p) & =-\int \frac{\mathrm{d}^{D} k}{(2 \pi)^{D}} \frac{1}{(p-k)^{2}-m_{1}^{2}+i \epsilon} \frac{1}{-k^{2}+m_{2}^{2}+i \epsilon} \\
& =-\int_{0}^{1} \mathrm{~d} x \int \frac{\mathrm{d}^{D} k}{(2 \pi)^{D}} \frac{1}{\left[i \epsilon-(1-2 x) k^{2}+p^{2} \frac{x(1-x)}{1-2 x}-m_{1}^{2} x+m_{2}^{2}(1-x)\right]^{2}},
\end{aligned}
$$

where $D$ denotes the continued spacetime dimension. At this point, we break the $x$ integral into the sum of two pieces, the integral on $0 \leqslant x \leqslant 1 / 2$ and the integral on $1 / 2 \leqslant x \leqslant 1$. The two are defined by opposite $i \epsilon$ prescriptions, since the coefficients of $k^{2}$ have opposite signs.

We can check the method in the simple case $m_{2}=0, m_{1}=m$, where we get, after expanding around $D=4$,

$$
\begin{aligned}
i(4 \pi)^{2} \Sigma^{\prime}(p)= & -\left[\frac{2}{4-D}+2-\gamma_{E}+\ln (4 \pi)\right] \frac{m^{2} p^{2}}{\left(p^{2}\right)^{2}+\epsilon^{2}}+\frac{1}{2} \ln \left(\left(p^{2}\right)^{2}+\epsilon^{2}\right) \\
& +\frac{m^{2} \ln m^{2}}{p^{2}-i \epsilon}-\frac{m^{2}}{2}\left[\frac{\ln \left(p^{2}+i \epsilon\right)}{p^{2}+i \epsilon}+\frac{\ln \left(p^{2}-i \epsilon\right)}{p^{2}-i \epsilon}\right] \\
& -\frac{p^{2}-m^{2}+i \epsilon}{p^{2}+i \epsilon} \ln \left(p^{2}-m^{2}+i \epsilon\right) .
\end{aligned}
$$


This result agrees with (3.1) for $p^{2}>0$ upon the cutoff identification

$$
\ln \Lambda^{2}=\frac{2}{4-D}+2-\gamma_{E}+\ln \pi
$$

With generic masses, we proceed as follows. After breaking the $x$ integral into the sum of the integrals on $0 \leqslant x \leqslant 1 / 2$ and $1 / 2 \leqslant x \leqslant 1$, we rescale $\epsilon$ and convert the second integral into another integral on $0 \leqslant x \leqslant 1 / 2$ by means of the change of variables $x \rightarrow 1-x$. So doing, we get

$$
\Sigma^{\prime}(p)=U\left(p^{2}+i \epsilon, m_{1}^{2}, m_{2}^{2}\right)-U\left(p^{2}-i \epsilon, m_{2}^{2}, m_{1}^{2}\right)
$$

where

$$
U(a, b, c)=\frac{i \Gamma\left(\frac{4-D}{2}\right)}{(4 \pi)^{D / 2}} \int_{0}^{1 / 2} \mathrm{~d} x(1-2 x)^{2-D}[a x(1-x)-(1-2 x)(b x-c(1-x))]^{(D-4) / 2} .
$$

The $x$ integration is relatively straightforward and, after the expansion around $D=4$, we obtain

$$
i(4 \pi)^{2} U\left(p^{2}, m_{1}^{2}, m_{2}^{2}\right)=\frac{v_{+}}{2 p^{2}}\left(\ln \frac{4 \Lambda^{2}}{m_{2}^{2}}-z \ln \frac{1+z}{1-z}\right),
$$

where $\ln \Lambda^{2}$ is defined as in (3.3) and

$$
z=\frac{\sqrt{u_{+} u_{-}}}{v_{+}}, \quad u_{ \pm}=\left(m_{1} \pm m_{2}\right)^{2}-p^{2}, \quad v_{ \pm}=p^{2} \mp m_{1}^{2} \pm m_{2}^{2} .
$$

Since $\Sigma^{\prime}$ turns into its complex conjugate under the replacement $m_{1} \leftrightarrow m_{2}$, we can assume, with no loss of generality, that $m_{1}>m_{2}$. If we define

$$
x=\frac{\sqrt{\left|u_{+} u_{-}\right|}}{v_{+}}, \quad y=\frac{\sqrt{\left|u_{+} u_{-}\right|}}{v_{-}}, \quad z^{\prime}=\frac{\sqrt{u_{+} u_{-}}}{v_{-}},
$$

we find the table

\begin{tabular}{|c|c|c|c|}
\hline$p^{2}$ range & $\left.z \ln \frac{1+z}{1-z}\right|_{p^{2} \rightarrow p^{2}+i \epsilon}$ & $\left.z^{\prime} \ln \frac{1+z^{\prime}}{1-z^{\prime}}\right|_{p^{2} \rightarrow p^{2}-i \epsilon}$ & \\
\hline$u_{+}<0$ & $x \ln \left(\frac{1+x}{1-x}\right)$ & $y \ln \left(\frac{1+y}{1-y}\right)$ & $0<x<1,0<y<1$ \\
$-v_{+}<0<u_{+}$ & $-2 x \arctan (x)$ & $-2 y \arctan (y)$ & $x>0, y>0$ \\
$u_{-}<0<-v_{+}$ & $-2 x \arctan (x)-2 \pi x$ & $-2 y \arctan (y)$ & $x<0, y>0$ \\
$-p^{2}<0<u_{-}$ & $x \ln \left(\frac{1+x}{1-x}\right)-2 i \pi x$ & $y \ln \left(\frac{1+y}{1-y}\right)$ & $-1<x<0,0<y<1$ \\
$-v_{-}<0<-p^{2}$ & $x \ln \left(\frac{1+x}{x-1}\right)-i \pi x$ & $y \ln \left(\frac{1+y}{y-1}\right)+i \pi y$ & $x<-1, y>1$ \\
$0<-v_{-}$ & $x \ln \left(\frac{1+x}{x-1}\right)-i \pi x$ & $y \ln \left(\frac{1+y}{y-1}\right)+i \pi y$ & $x<-1, y<-1$ \\
\hline
\end{tabular}


Finally, if we use (6.1) for $\Sigma(p)$, formula (2.2) gives

$$
\Sigma_{\mathrm{FW}}(p)=\frac{1}{2}\left(V\left(p^{2}+i \epsilon, m_{1}^{2}, m_{2}^{2}\right)+U\left(p^{2}+i \epsilon, m_{1}^{2}, m_{2}^{2}\right)-U\left(p^{2}-i \epsilon, m_{2}^{2}, m_{1}^{2}\right)\right),
$$

where $V(a, b, c)$ is given by formula (6.2) of section 6 . In the case of $\Sigma_{\text {FW-FW }}$, formula (2.8) gives

$$
\begin{aligned}
\Sigma_{\mathrm{FW}-\mathrm{FW}}(p)= & \frac{1}{4}\left(V\left(p^{2}+i \epsilon, m_{1}^{2}, m_{2}^{2}\right)+U\left(p^{2}+i \epsilon, m_{1}^{2}, m_{2}^{2}\right)-U\left(p^{2}-i \epsilon, m_{2}^{2}, m_{1}^{2}\right)\right. \\
& \left.-V\left(p^{2}-i \epsilon, m_{1}^{2}, m_{2}^{2}\right)-U\left(p^{2}-i \epsilon, m_{1}^{2}, m_{2}^{2}\right)+U\left(p^{2}+i \epsilon, m_{2}^{2}, m_{1}^{2}\right)\right) .
\end{aligned}
$$

Now we analyze the meaning of these results.

\section{Unitarity}

In this section, we study the imaginary parts of the amplitudes and show that the theory propagating FW particles is not consistent with the optical theorem.

The optical theorem is another way to express the unitarity of the $S$ matrix. Writing $S=1+i T$, the identity $S^{\dagger} S=1$ becomes $-i T+i T^{\dagger}=T^{\dagger} T$ and can be rephrased diagrammatically by means of cut diagrams, which are diagrams divided into two parts by a cut that crosses internal legs $[17,23,24]$. One side of the cut is due to the factor $T$ of $T^{\dagger} T$. There, the diagram is unshadowed and the vertices and propagators are those given by the usual Feynman rules (once the usual factors of $i$ are restored in vertices and propagators). The other side of the cut is due to the factor $T^{\dagger}$ of $T^{\dagger} T$. It is shadowed and the vertices and propagators are the complex conjugates of those given by the Feynman rules. Finally, "cut propagators" account for the legs crossed by the cut. The cut lines represent the product of $T^{\dagger}$ and $T$ in $T^{\dagger} T$.

The cut diagrams encode the imaginary part of the amplitude and the cross section for the production of the particles circulating in the loop. This means that, beyond appropriate thresholds, the virtual particles of the loop may turn into real particles.

Let us start from the usual bubble diagram $\Sigma(p)$, where both propagators are defined by means of the Feynman prescription. There, the true threshold is $p^{2}=\left(m_{1}+m_{2}\right)^{2}$ (i.e. $\left.u_{+}=0\right)$ and above it, the imaginary part $\operatorname{Im}(-i \Sigma)$ is nontrivial. From formula (6.4) of section 6 we find

$$
2 \operatorname{Im}(-i \Sigma)=\frac{\sqrt{u_{+} u_{-}}}{8 \pi p^{2}} \theta\left(-u_{+}\right) .
$$

This result obeys the optical theorem, which states in this case that $2 \operatorname{Im}(-i \Sigma)$ is equal to the sum of the cut diagrams shown in figure 1, where the cut propagator is the top one of figure 2. It means that for $p^{2}>\left(m_{1}+m_{2}\right)^{2}$ the incoming particle can decay into the virtual particles circulating in the loop, which are then turned into real particles.

For future use, we recall that one cut diagram is obtained by replacing the two internal propagators of $\Sigma(p)$ by the cut propagators

$$
(2 \pi) \theta\left(p^{0}-k^{0}\right) \delta\left((p-k)^{2}-m_{1}^{2}\right), \quad(2 \pi) \theta\left(k^{0}\right) \delta\left(k^{2}-m_{2}^{2}\right) .
$$

The other cut diagram is obtained by flipping the signs of the arguments of the $\theta$ functions. 


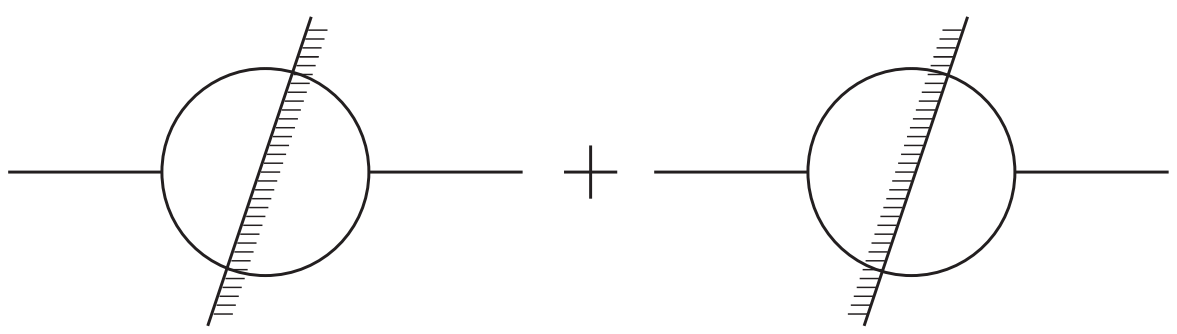

Figure 1. Cut diagrams that give the imaginary part of $-2 i$ times the bubble diagram.

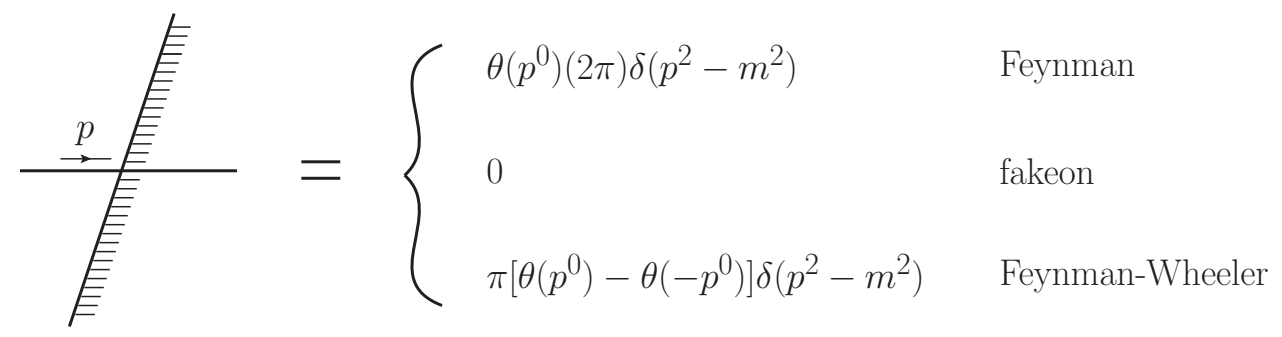

Figure 2. Cut propagators in the various cases.

The Landau equations [25] provide a systematic method to identify the potential thresholds of a loop integral, where the amplitude may be nonanalytic. It is well known that in the case of $\Sigma(p)$ the Landau equations give a second potential threshold, which is $p^{2}=\left(m_{1}-m_{2}\right)^{2}$ (i.e. $u_{-}=0$ ). However, that threshold is not associated with any pinching singularities of the integral $\Sigma(p)$, which means that it is not a true threshold. For this reason, it is commonly called pseudothreshold.

When we consider $\Sigma^{\prime}(p)$, we find that the two potential thresholds exchange roles and $p^{2}=\left(m_{1}-m_{2}\right)^{2}$ becomes the true threshold, while $p^{2}=\left(m_{1}+m_{2}\right)^{2}$ becomes a pseudothreshold. Moreover, the imaginary part of $-i \Sigma^{\prime}$ is nonvanishing below its true threshold, which means for $p^{2}<\left(m_{1}-m_{2}\right)^{2}$, not above it. Specifically, using (3.8), formulas (3.4) and (3.6) allow us to derive

$$
2 \operatorname{Im}\left(-i \Sigma^{\prime}\right)=-\frac{\sqrt{u_{+} u_{-}}}{8 \pi p^{2}} \theta\left(u_{-}\right) .
$$

The troubles with FW particles are evident from this formula. Indeed, taking $\Sigma^{\prime}=i T$, the optical theorem $-i T+i T^{\dagger}=T^{\dagger} T$ interprets the left-hand side of (4.3) as a forward scattering amplitude and implies that the right-hand side is the total cross section for the production of the virtual-turned-into-real particles in all final states. In particular, since $T^{\dagger} T \geqslant 0$, the right-hand side of (4.3) should be nonnegative, which is evidently false for $p^{2}>0$. Basically, (4.3) says that at $p^{2}>0$ an incoming particle has a negative probability to decay into the final particles! Moreover, (4.3) is singular for $p^{2}=0$, which means that for $p^{2} \rightarrow 0^{ \pm}$the cross section is infinite. Such a singularity is clearly absent in the usual case, as formula (4.1) shows.

The result (4.3) also admits an interpretation in terms of cut diagrams. Precisely, for $p^{2}>0$ the right-hand side of (4.3) is still equal to the sum of the cut diagrams of figure 1 , 
but the left diagram has cut propagators

$$
(2 \pi) \theta\left(p^{0}-k^{0}\right) \delta\left((p-k)^{2}-m_{1}^{2}\right), \quad-(2 \pi) \theta\left(-k^{0}\right) \delta\left(k^{2}-m_{2}^{2}\right),
$$

while the right diagram is obtained from the left one by flipping the signs of the arguments of the $\theta$ functions.

The important point is that the sign of the argument of second $\theta$ function of formula (4.4) is reversed with respect to the one of (4.2), which leads to instability (see the next section).

The result is confirmed at $p^{2}<0$, but with a caveat. Indeed, (4.3) is positive for $p^{2}<0$, but the cut diagram with propagators (4.4) looks negative for both $p^{2}>0$ and $p^{2}<0$. The reason why there is no contradition is that the cut diagrams give integrals that are divergent for $p^{2}<0$, so the final result may have an unexpected sign. If we use the cutoff $\Lambda$ on the $k_{s}$ integral, it is easy to check (choosing e.g. $p^{0}=0$ ) that the resul is (4.3) plus the linear divergence met in formula (2.7). If we use the dimensional technique, the linear divergences are set automatically to zero and we just find (4.3).

Diverging imaginary parts are quite unusual. However, they should be expected when both prescriptions $\pm i \epsilon$ are present in the same diagram, since in ref. [9] it was found that both the locality and hermiticity of counterterms are violated in such cases.

In the end, we do have a negative $\operatorname{Im}\left(-i \Sigma^{\prime}\right)$ for $p^{2}>0$, which means that the theory can be at most pseudounitary, but not unitary. Since the right-hand side of the identity $-i T+i T^{\dagger}=T^{\dagger} T$ cannot be positive definite, it should at best be replaced by $T^{\dagger} H T$, where $H$ is a matrix that has both positive and negative eigenvalues. However, pseudounitarity is not a viable concept for a physically meaningful theory.

In total, the imaginary part of $-2 i \Sigma_{\mathrm{FW}}$ follows from formula (3.9) and is equal to

$$
2 \operatorname{Im}\left(-i \Sigma_{\mathrm{FW}}\right)=\frac{\sqrt{u_{+} u_{-}}}{16 \pi p^{2}}\left[\theta\left(-u_{+}\right)-\theta\left(u_{-}\right)\right] .
$$

It can be obtained as the sum of the cut diagrams of figure 1 with one cut propagator given by the top line of figure 2 and the other cut propagator given by the bottom line of that figure. Explicitly, one cut diagram has propagators

$$
(2 \pi) \theta\left(p^{0}-k^{0}\right) \delta\left((p-k)^{2}-m_{1}^{2}\right), \quad \operatorname{sgn}\left(k^{0}\right) \pi \delta\left(k^{2}-m_{2}^{2}\right),
$$

and the other one is obtained by flipping the signs of the arguments of the $\theta$ and sign functions.

If the FW particles were purely virtual, their cut propagator and $\operatorname{Im}\left(-i \Sigma_{\mathrm{FW}}\right)$ would have to vanish, to be consistent with the optical theorem. Indeed, we know that the cut diagrams are related to the cross section for the production of the particles circulating in the loop. Purely virtual particles can never be produced, by definition. Since $\operatorname{Im}\left(-i \Sigma_{\mathrm{FW}}\right)$ is not zero, we must conclude that a FW particle is not purely virtual. Moreover, $\operatorname{Im}\left(-i \Sigma_{\mathrm{FW}}\right)$ is nonvanishing even for $p^{2}<\left(m_{1}-m_{2}\right)^{2}$. This result is difficult to interpret, since it amounts to the production of particles with negative energies and leads to instability (see the next section). 
Note that formula (2.8) gives the extremely simple result

$$
\Sigma_{\mathrm{FW}-\mathrm{FW}}(p)=-\operatorname{Im}\left(-i \Sigma_{\mathrm{FW}}\right)=-\frac{\sqrt{u_{+} u_{-}}}{32 \pi p^{2}}\left[\theta\left(-u_{+}\right)-\theta\left(u_{-}\right)\right],
$$

which means that the amplitude $-i \Sigma_{\mathrm{FW}-\mathrm{FW}}$ is purely imaginary. Indeed, by definition $\Sigma_{\mathrm{FW}-\mathrm{FW}}$ is real. Consistently with what we have found above, $2 \operatorname{Im}\left(-i \Sigma_{\mathrm{FW}-\mathrm{FW}}\right)$ is equal to the sum of the two cut diagrams of figure 1 with the cut propagators given by the third line of figure 2 .

From the result (4.7) we see that the FW propagators break analyticity in an unusual way. In section 6 we will see that, instead, fakeons upgrade the common notion of analyticity to the more general notion of regionwise analyticity.

In ref. [7] the imaginary part of $\Sigma_{\mathrm{FW}}(p)$ is claimed to be just one half the imaginary part of the usual bubble diagram $\Sigma(p)$, since the authors miss the contribution from $\Sigma^{\prime}(p)$, which is the piece proportional to $\theta\left(u_{-}\right)$in formula (4.5).

\section{Stability}

In this section we show that a theory propagating FW particles violates stability. Specifically, the denominators of (2.3) and (2.5) vanish for

$$
\left|p^{0}\right|=\omega_{1}-\omega_{2}
$$

These singularities tell us where $\Sigma^{\prime}(p)$ may be nonanalytic, which in the case at hand means that it has branch cuts. The discontinuity of the amplitude around the cuts leads to the nontrivial imaginary part $\operatorname{Im}\left(-i \Sigma^{\prime}\right)$. As we know, the optical theorem relates the discontinuity to a physical process that turns the circulating virtual particles into real particles.

The crucial aspect of formula (5.1) is that the external energy is equated to a difference of frequencies, rather than a sum. Thus, if we assume $p^{0}>0$, for definiteness, the physical process associated with $\operatorname{Im}\left(-i \Sigma^{\prime}\right)$ is an external particle of momentum $p$ decaying into a particle of energy $\omega_{1}$ and a particle of energy $p^{0}-\omega_{1}=-\omega_{2}$. This means instability, because the energies of the final particles are unbounded.

Explicitly, for large momentum $k_{s}$, we have

$$
p^{0}=\omega_{1}-\omega_{2} \sim-p_{s} \cos \theta+\frac{1}{2 k_{s}}\left[m_{1}^{2}-m_{2}^{2}+p_{s}^{2} \sin ^{2} \theta\right]+\mathcal{O}\left(\frac{1}{k_{s}^{2}}\right) .
$$

Let us assume $m_{1}>m_{2}$ and take, for example, $\theta=\pi / 2$. Then (5.2) has solutions for arbitrarily large $k_{s}$ and arbitrarily small incoming energies $p^{0}$. If the incoming particle is massless, or has a very small mass, it can decay into particles with arbitrarily large positive and negative energies. Moreover, as we know from the divergent behaviors of formulas (4.3), (4.5) and (4.7) at $p^{2}=0$, the probability of such an occurrence is huge and negative. This is an unacceptable dynamics and another reason why the FW propagator is not good for Feynman diagrams. 


\section{$6 \quad$ Fakeons as purely virtual quanta}

In this section we explain how the fakeons avoid the problems of the Feynman-Wheeler particles and provide the correct concept of purely virtual quanta.

The calculations of diagrams with fakeons require crucial modifications with respect to the calculations of diagrams with Feynman propagators. For this reason, it is convenient to briefly revisit the standard bubble diagram $\Sigma(p)$. Using Feynman parameters, the loop integral

$$
\Sigma(p)=\int \frac{\mathrm{d}^{D} k}{(2 \pi)^{D}} \frac{1}{(p-k)^{2}-m_{1}^{2}+i \epsilon} \frac{1}{k^{2}-m_{2}^{2}+i \epsilon}
$$

gives

$$
\Sigma(p)=V\left(p^{2}+i \epsilon, m_{1}^{2}, m_{2}^{2}\right)
$$

where

$$
V(a, b, c)=\frac{i \Gamma\left(\frac{4-D}{2}\right)}{(4 \pi)^{D / 2}} \int_{0}^{1} \mathrm{~d} x[-a x(1-x)+b x+c(1-x)]^{(D-4) / 2} .
$$

Defining $u_{ \pm}$as in (3.7) and expanding around $D=4$, we obtain

$i(4 \pi)^{2} V\left(p^{2}, m_{1}^{2}, m_{2}^{2}\right)=-\ln \frac{4 \Lambda^{2}}{m_{1} m_{2}}+\frac{m_{1}^{2}-m_{2}^{2}}{p^{2}} \ln \frac{m_{1}}{m_{2}}-\frac{\sqrt{u_{+} u_{-}}}{p^{2}} \ln \frac{m_{1}^{2}+m_{2}^{2}-p^{2}+\sqrt{u_{+} u_{-}}}{2 m_{1} m_{2}}$,

where $\ln \Lambda^{2}$ is defined again as in (3.3).

Making (6.3) more explicit, we find [26-28]

$$
\begin{array}{rl}
i(4 \pi)^{2} & V\left(p^{2}+i \epsilon, m_{1}^{2}, m_{2}^{2}\right)=-\ln \frac{4 \Lambda^{2}}{m_{1} m_{2}}+\frac{m_{1}^{2}-m_{2}^{2}}{p^{2}} \ln \frac{m_{1}}{m_{2}} \\
& -\frac{\sqrt{u_{+} u_{-}}}{p^{2}} \theta\left(u_{-}\right)\left(\ln \frac{\sqrt{u_{+}}+\sqrt{u_{-}}}{\sqrt{u_{+}}-\sqrt{u_{-}}}\right)+\frac{2 \sqrt{-u_{+} u_{-}}}{p^{2}} \theta\left(-u_{-}\right) \theta\left(u_{+}\right) \arctan \sqrt{\frac{-u_{-}}{u_{+}}} \\
& +\frac{\sqrt{u_{+} u_{-}}}{p^{2}} \theta\left(-u_{+}\right)\left(\ln \frac{\sqrt{-u_{-}}+\sqrt{-u_{+}}}{\sqrt{-u_{-}}-\sqrt{-u_{+}}}-i \pi\right) .
\end{array}
$$

To explain how to proceed when the bubble diagram involves circulating fakeons, we start from the case where one internal leg is a fakeon and the other internal leg is a Feynman propagator. The loop integral reads

$$
\left.\Sigma_{\mathrm{f}}(p) \equiv \int \frac{\mathrm{d}^{D} k}{(2 \pi)^{D}} \frac{1}{(p-k)^{2}-m_{1}^{2}+i \epsilon} \frac{1}{k^{2}-m_{2}^{2}}\right|_{\mathrm{f}},
$$

where the fakeon prescription is denoted by means of the subscript $f$.

The simplest way to formulate the fakeon prescription is to make the Wick rotation from the Euclidean version of the diagram and complete it nonanalytically by means of an operation called average continuation $[11,29]$, which we describe below.

Specifically, we start from the Euclidean version of the diagram $\Sigma(p)$ and initiate the Wick rotation, i.e. move analytically from the Euclidean region, where the energies are purely imaginary, to the Minkowskian region. So doing, the thresholds we find on the real axis coincide with the ones of $\Sigma(p)$. In particular, the true threshold is still $p^{2}=\left(m_{1}+m_{2}\right)^{2}$, 
while $p^{2}=\left(m_{1}-m_{2}\right)^{2}$ is a pseudothreshold. Below the threshold $p^{2}=\left(m_{1}+m_{2}\right)^{2}$ we find no obstacle and conclude the Wick rotation as usual. This means that the loop integrals $\Sigma(p)$ and $\Sigma_{\mathrm{f}}(p)$ coincide and are analytic there:

$$
\Sigma_{\mathrm{f}}(p)=\Sigma(p) \quad \text { for } p^{2}<\left(m_{1}+m_{2}\right)^{2} .
$$

Note that the fact that the pseudothreshold does not behave differently from usual is crucial for stability, since kinematic relations like (5.1) do not play any role.

Above the threshold $p^{2}=\left(m_{1}+m_{2}\right)^{2}$ the difference between $\Sigma_{\mathrm{f}}(p)$ and $\Sigma(p)$ becomes apparent. In the case of $\Sigma(p)$, the threshold is crossed analytically by means of the Feynman prescription $p^{2} \rightarrow p^{2}+i \epsilon$. This means that the Wick rotation is completed analytically. In the case of $\Sigma_{\mathrm{f}}(p)$ the threshold is crossed by means of the fakeon prescription, which amounts to taking the arithmetic average of the two analytic continuations around the threshold, which correspond to the prescriptions $p^{2} \rightarrow p^{2} \pm i \epsilon$. This operation, called average continuation [11, 29], is unambiguous, but not analytic. Hence, we speak about nonanalytic Wick rotation [29]. Despite being nonanalytic, it returns an analytic function above the threshold. What is not analytic is just the relation between the two analytic functions that encode the amplitude below and above the threshold.

We know that $\Sigma(p)$ is given by formula (6.4). When we replace a particle circulating in the loop with a fakeon, the only difference is that the $i \pi$ of the last line disappears. Indeed, the $i \pi$ of (6.4) is due to having crossed the threshold by means of the analytic continuation $p^{2} \rightarrow p^{2}+i \epsilon$. The other analytic continuation $p^{2} \rightarrow p^{2}-i \epsilon$ gives $-i \pi$, so the arithmetic average of the two gives zero. At the end, equation (6.5) extends to

$$
\begin{aligned}
\Sigma_{\mathrm{f}}(p) & =\frac{1}{2}\left(V\left(p^{2}+i \epsilon, m_{1}^{2}, m_{2}^{2}\right)+V\left(p^{2}-i \epsilon, m_{1}^{2}, m_{2}^{2}\right)\right) \\
& =\frac{1}{2}\left(\Sigma(p)-\Sigma^{*}(p)\right)=\Sigma(p)+\frac{\sqrt{u_{+} u_{-}}}{16 \pi p^{2}} \theta\left(-u_{+}\right) .
\end{aligned}
$$

In particular,

$$
2 \operatorname{Im}\left(-i \Sigma_{\mathrm{f}}\right)=0 .
$$

This result is consistent with the optical theorem. We know that the discontinuity of the amplitude above the threshold, which is encoded in its imaginary part, is associated with a physical process where the particles circulating in the loop become real. However, a purely virtual quantum cannot become real, by definition. This means that when fakeons are involved such a process has zero chances to occur, i.e. the imaginary part vanishes above the threshold. Then the potential physical process becomes a fake process.

If we interpret (6.7) in terms of the cutting equations, so that $2 \operatorname{Im}\left(-i \Sigma_{\mathrm{f}}\right)$ equals the sum of the cut diagrams of figure 1, we can say that the cut propagator of the fakeon is identically zero, as shown in figure 2 .

Analyticity holds above $p^{2}=\left(m_{1}+m_{2}\right)^{2}$ in both cases $\Sigma$ and $\Sigma_{\mathrm{f}}$, but in different senses. In the case of $\Sigma_{\mathrm{f}}$, the space of complexified external momenta $p$ is divided into two disjoint regions of analyticity. One region is located below the threshold and the other one is located above the threshold. The former is the Euclidean region, the latter is a fakeon region. 
Specifically, if we take formula (6.4) and drop the $i \pi$ appearing in the last line, we have an analytic function for $p^{2}<\left(m_{1}+m_{2}\right)^{2}$ and another analytic function for $p^{2}>\left(m_{1}+m_{2}\right)^{2}$. The two are not analytically related to each other. However, the former unambiguously determines the latter by means of the average continuation.

Note that, in general, there is no way to determine the function below the threshold from the function above the threshold [11] (although in the particular case at hand it seems that we may achieve this goal). For example, in three spacetime dimensions we often meet the square-root function $\sqrt{z}$ ( $z$ being $-p^{2}-i \epsilon$ or a more complicated function of $\left.p^{2}\right)$. Its average continuation on the negative real axis is zero and obviously we cannot recover $\sqrt{z}$ from the zero function.

The new analyticity property of the amplitudes involving fakeons is called "regionwise analyticity".

Note that the fakeon prescription is free of nonlocal divergences. Indeed, the divergences coincide with those of $\Sigma(p)$, which are local.

If both particles circulating in the loop are fakeons, we need to compute the loop integral

$$
\left.\left.\Sigma_{\mathrm{ff}}(p) \equiv \int \frac{\mathrm{d}^{D} k}{(2 \pi)^{D}} \frac{1}{(p-k)^{2}-m_{1}^{2}}\right|_{\mathrm{f}} \frac{1}{k^{2}-m_{2}^{2}}\right|_{\mathrm{f}} .
$$

We can proceed as above, starting from the Euclidean framework and ending the Wick rotation nonanalytically by means of the average continuation above the threshold $p^{2}=$ $\left(m_{1}+m_{2}\right)^{2}$. The result is

$$
\Sigma_{\mathrm{ff}}(p)=\Sigma_{\mathrm{f}}(p)=\Sigma(p)+\frac{\sqrt{u_{+} u_{-}}}{16 \pi p^{2}} \theta\left(-u_{+}\right)
$$

and again $2 \operatorname{Im}\left(-i \Sigma_{\mathrm{ff}}\right)=0$.

We can summarize the crucial differences among the calculations of $\Sigma(p), \Sigma_{\mathrm{f}}(p)$, and $\Sigma_{\mathrm{FW}}(p)$ as follows. $\Sigma(p)$ can be computed directly in Minkowski spacetime or by means of the analytic Wick rotation from the Euclidean version of the loop integral; $\Sigma_{\mathrm{f}}(p)$ is computed from the Euclidean version, but the Wick rotation is completed nonanalytically by means of the average continuation; $\Sigma_{\mathrm{FW}}(p)$ is computed directly in Minkowski spacetime, since the principal-value prescription is inherently Minkowskian. The integral on the loop energy picks the same residues in the cases of $\Sigma(p)$ and $\Sigma_{\mathrm{f}}(p)$, but different residues in the case of $\Sigma_{\mathrm{FW}}(p)$.

In the end, the results obtained with the fakeon propagator are quite different from the ones due to the FW propagator. With fakeons: $(i)$ the optical theorem, hence unitarity, holds; (ii) no instability is generated, since the thresholds are the same as usual, which correspond to kinematics like

$$
\left|p^{0}\right|=\omega_{1}+\omega_{2}
$$

instead of (5.1); (iii) finally, there is no problem with the locality of counterterms, since the divergent part can be computed in the Euclidean region, where, by formula (6.5), $\Sigma_{\mathrm{f}}$ and $\Sigma$ coincide. Once the amplitude is renormalized there, its average continuation is renormalized everywhere, like its analytic continuation. 


\section{Lower dimensions}

Now we repeat the analysis of FW particles in three and two spacetime dimensions.

In three dimensions we assume $p^{2}>0$ and make the calculation by adapting formula (2.3). We obtain

$$
\Sigma^{\prime}(p)=\frac{i}{8 \pi \sqrt{p^{2}}} \ln \frac{\sqrt{p^{2}-i \epsilon}+m_{1}-m_{2}}{\sqrt{p^{2}+i \epsilon}-m_{1}+m_{2}} .
$$

The (negative) imaginary part

$$
2 \operatorname{Im}\left(-i \Sigma^{\prime}\right)=-\frac{\theta\left(u_{-}\right)}{4 \sqrt{p^{2}}}
$$

can be verified by evaluating the cut diagrams of figure 1 with the rules explained in section 4 .

In two dimensions it is more convenient to use the dimensional regularization and expand the result of (3.5) around $D=2$. We find

$$
U\left(p^{2}, m_{1}^{2}, m_{2}^{2}\right)=\frac{i}{4 \pi \sqrt{u_{+} u_{-}}} \ln \frac{1+z}{1-z} .
$$

Thus, using (3.8) the discontinuity of the amplitude is

$$
2 \operatorname{Im}\left(-i \Sigma^{\prime}\right)=-\frac{\theta\left(u_{-}\right)}{\sqrt{u_{+} u_{-}}},
$$

which is also negative. Again, the result can be verified by computing the cut diagrams of figure 1 with the rules of section 4 .

We see that the problems found in four dimensions are essentially confirmed in lower dimensions.

\section{Fakeons, God's energy and the infinite desert}

We point out that the fakeon prescription virtualizes a particle (or a ghost) completely and eradicates it from the theory. In particular, a theory with fakeons is as fundamental as the standard model.

If a theory has ghosts, it is not acceptable as a fundamental theory, even if the ghosts have a finite lifetime and decay. Indeed, an unstable particle or ghost is not really out of the physical spectrum, in the same way as the muon is not out of the physical spectrum of the standard model. "Living with ghosts" [30] is not a viable option, even if the ghosts may be unobservable in common settings.

The only wayout is to quantize the would-be ghosts in a radically different way, that is to say as fakeons. Then they are really out of the physical spectrum, at all energies.

The most important application of the fakeon prescription is that it allows us to make sense of quantum gravity as a perturbative quantum field theory $[10,31]$. The theory is 
described by the action

$$
S_{\mathrm{QG}}(g, \Phi)=-\frac{1}{2 \kappa^{2}} \int \mathrm{d}^{4} x \sqrt{-g}\left[2 \Lambda_{C}+\zeta R+\alpha\left(R_{\mu \nu} R^{\mu \nu}-\frac{1}{3} R^{2}\right)-\frac{\xi}{6} R^{2}\right]+S_{\mathfrak{m}}(g, \Phi),
$$

where $\alpha, \xi, \zeta$ and $\kappa$ are positive constants, $M_{\mathrm{Pl}}=1 / \sqrt{G}=\sqrt{8 \pi \zeta} / \kappa$ is the Planck mass, $\Phi$ are the matter fields and $S_{\mathfrak{m}}$ is the action of the matter sector. Besides the graviton, the theory propagates two massive fields: a scalar $\phi$ and a spin-2 field $\chi_{\mu \nu}$. The residue of the $\chi_{\mu \nu}$ free propagator has the wrong sign, so $\chi_{\mu \nu}$ must be quantized as a fakeon, because the Feynman prescription would turn it into a ghost. The residue at the $\phi$ pole has the correct sign, so $\phi$ can be quantized either as a fakeon or a true particle. The masses $m_{\phi}=\sqrt{\zeta / \xi}$ and $m_{\chi}=\sqrt{\zeta / \alpha}$ (neglecting small corrections due to the cosmological constant $\Lambda_{C}$ ) are free parameters and should be determined experimentally. Their values could be smaller, or even much smaller, than the Planck mass, e.g. $m_{\phi} \sim m_{\chi} \sim 10^{12} \mathrm{GeV}$. The fine structure constants that govern the perturbative expansion of (8.1) are the ratios $\alpha_{\chi}=m_{\chi}^{2} / M_{\mathrm{Pl}}^{2}$ and $\alpha_{\phi}=m_{\phi}^{2} / M_{\mathrm{Pl}}^{2}$ and could be as small as $10^{-14}[32]$.

These facts imply that the pure gravitational sector of the theory is perturbative up to an unbelievably high energy. Indeed, since the action (8.1) is renormalizable, the perturbative expansion is not governed by the ratio $E / M_{\mathrm{Pl}}$, where $E$ is the center-of-mass energy, but by the running couplings $\alpha_{\chi}$ and $\alpha_{\phi}$. The running, in turn, is governed by logarithmic corrections. It takes a long way to turn the products

$$
\alpha_{\chi} \ln \frac{E^{2}}{\mu^{2}}, \quad \alpha_{\phi} \ln \frac{E^{2}}{\mu^{2}},
$$

into quantities of order 1 , whatever reference energy $\mu$ we take. Precisely, $\alpha_{\chi} \sim \alpha_{\phi} \sim 10^{14}$ and $\mu \sim M_{\mathrm{Pl}}$ give the unbelievably high energy

$$
E \sim 10^{10^{13}} M_{\mathrm{Pl}}
$$

which we think deserves to be called "God's energy" for this reason. If we could multiply the maximum energy we can reach in our laboratories by a factor 10 every year, we would reach the Planck scale in less than twenty years and God's energy in one thousand times the age of the universe.

Note that small couplings $\alpha_{\chi}$ and $\alpha_{\phi}$ do not mean that the theory is practically free. For example, $\phi$ and $\chi_{\mu \nu}$ have widths that are proportional to their masses times $\alpha_{\phi}$ and $\alpha_{\chi}$, respectively [32]. Since the masses are large, we obtain nonnegligible widths even if $\alpha_{\phi}$ and $\alpha_{\chi}$ are small. Actually, the width of $\chi_{\mu \nu}$ is comparable to the widths of the $Z$ and $W$ bosons and the one of the Higgs boson $H$.

Of course, when we say that the theory is perturbative up to God's energy, we refer to the elementary processes (graviton-graviton scattering, graviton-matter scattering, etc.). Nonperturbative problems are present at all energies, when they involve large numbers of particles and gravitons at the same time, as in the classical limit, black holes, etc.

The quantum gravity theory based on the fakeon idea predicts new physics below the Planck scale (precisely, at energies equal to the masses of $\phi$ and $\chi$ ) and then at the Planck 
scale itself. At the same time, it opens up an extremely alarming scenario, which is the threat of an "infinite desert" from the Planck scale to God's energy: no new physics, nothing interesting, forever! Although we can always assume that extra, very heavy particles and/or fakeons exist in such a huge range of energies without affecting any fundamental principles, this kind of variety might be rather unexciting.

Long ago Weinberg proposed asymptotic safety as a way to overcome the nonrenormalizability of Einstein gravity [33]. The idea relies on the assumption that the ultraviolet limit is an interacting conformal fixed point with a finite-dimensional critical surface. In Weinberg's approach one has to advocate nonperturbative or semi-nonperturbative methods, which make it difficult to discuss the issue of unitarity. Moreover, assumptions on the ultraviolet limit are hard to accept, given that it is experimentally out of reach, by definition.

A related issue is ultraviolet completeness, which is defined in different ways and sometimes linked to asymptotic safety or asymptotic freedom, although such notions do not appear to be necessary requirements for completeness. We emphasize that the theory based on the fakeon idea (which is not asymptotically free $[31]^{2}$ ) is safe enough and ultraviolet complete enough, due to its huge perturbative regime. Finally, its nonperturbative sector is candidate to explain even what lies beyond God's energy. In this sense, it can be considered ultraviolet complete.

\section{Conclusions}

The possibility that purely virtual entities might exist in nature is interesting in itself and has attracted the attention of several scientists in the past, both at the classical and quantum levels. However, the correct candidate was not identified right away. At the classical level, Dirac virtualized runaway solutions by renouncing causality at small distances, while Feynman and Wheeler considered T-symmetric wave emissions in classical electrodynamics. At the quantum level, Bollini and Rocca picked up on the suggestion of Feynman and Wheeler and studied the Cauchy principal value as a propagator in Feynman diagrams.

For a variety of reasons, having both prescriptions $\pm i \epsilon$ in the same diagram is extremely dangerous, because it leads to violations of the locality of counterterms, unitarity and stability. The right purely virtual quanta turn out to be the fakeons, which can be used to virtualize both ghosts and normal particles. We have compared the Feynman, fakeon and Feynman-Wheeler prescriptions in the bubble diagram and studied the optical theorem in four, three and two dimensions. Only the fakeon has an identically vanishing cut propagator, which means that it remains virtual once the quantum corrections are turned on.

Fakeons allow us to make sense of quantum gravity at the fundamental level, and place that theory on an equal footing with the standard model. The theory of quantum gravity

\footnotetext{
${ }^{2}$ The divergent parts calculated with the Feynman and/or fakeon quantization prescriptions coincide with those of the Euclidean version of the theory [11]. The one-loop beta functions are also computed in $[34-36]$.
} 
that emerges from the fakeon idea is perturbative up to an unbelievably high energy, which we named God's energy. This opens up a frightening scenario: the possibility of an infinite desert between the Planck scale and God's energy, with no new physics in sight, basically forever.

\section{Acknowledgments}

I am grateful to U. Aglietti for helpful discussions. I also thank the participants of the conferences "Quantum gravity and quantum geometry", 3 Radboud University, Nijmegen, Oct.-Nov., 2019, and "Cosmological frontiers in fundamental physics 2019", ${ }^{4}$ Sept. 2019, Perimeter Institute, for valuable exchanges.

Open Access. This article is distributed under the terms of the Creative Commons Attribution License (CC-BY 4.0), which permits any use, distribution and reproduction in any medium, provided the original author(s) and source are credited.

\section{References}

[1] P.A.M. Dirac, Classical theory of radiating electrons, Proc. Roy. Soc. Lond. A 167 (1938) 148 [INSPIRE].

[2] J.D. Jackson, Classical electrodynamics, John Wiley and Sons, Inc. (1975), chapter 17.

[3] L. Bel and H.S. Zia, Regular reduction of relativistic theories of gravitation with a quadratic Lagrangian, Phys. Rev. D 32 (1985) 3128 [INSPIRE].

[4] L. Parker and J.Z. Simon, Einstein equation with quantum corrections reduced to second order, Phys. Rev. D 47 (1993) 1339 [gr-qc/9211002] [INSPIRE].

[5] J.A. Wheeler and R.P. Feynman, Interaction with the absorber as the mechanism of radiation, Rev. Mod. Phys. 17 (1945) 175.

[6] J.A. Wheeler and R.P. Feynman, Classical electrodynamics in terms of direct interparticle action, Rev. Mod. Phys. 21 (1949) 425 [inSPIRE].

[7] C.G. Bollini and M.C. Rocca, The Wheeler propagator, Int. J. Theor. Phys. 37 (1998) 2877 [hep-th/9807010] [INSPIRE].

[8] A. Plastino and M.C. Rocca, Quantum Field Theory, Feynman-, Wheeler Propagators, Dimensional Regularization in Configuration Space and Convolution of Lorentz Invariant Tempered Distributions, J. Phys. Comm. 2 (2018) 115029 [arXiv:1708.04506] [INSPIRE].

[9] U.G. Aglietti and D. Anselmi, Inconsistency of Minkowski higher-derivative theories, Eur. Phys. J. C 77 (2017) 84 [arXiv:1612.06510] [inSPIRE] and online at http://renormalization.com/16a2/.

[10] D. Anselmi, On the quantum field theory of the gravitational interactions, JHEP 06 (2017) 086 [arXiv: 1704.07728] [INSPIRE] and online at http://renormalization.com/17a3/.

[11] D. Anselmi, Fakeons And Lee-Wick Models, JHEP 02 (2018) 141 [arXiv:1801.00915] [INSPIRE] and online at http://renormalization.com/18a1/.

\footnotetext{
${ }^{3}$ http://www.noncommutativegeometry.nl/qgqg2019/.

${ }^{4}$ https://www.perimeterinstitute.ca/conferences/cosmological-frontiers-fundamental-physics-2019.
} 
[12] D. Anselmi, Fakeons and the classicization of quantum gravity: the FLRW metric, JHEP 04 (2019) 061 [arXiv:1901.09273] [INSPIRE] and online at http://renormalization.com/19a1/.

[13] T. Gaumnitz et al., Streaking of 43-attosecond soft-X-ray pulses generated by a passively CEP-stable mid-infrared driver, Opt. Exp. 25 (2017) 27506.

[14] J. Li et al., 53-attosecond X-ray pulses reach the carbon K-edge, Nat. Commun. 8 (2017) 186.

[15] D. Anselmi and A. Marino, Fakeons and Microcausality: Light Cones, Gravitational Waves and the Hubble Constant, accepted manuscript in Class. Quant. Grav. 21 February 2020, arXiv:1909.12873 [INSPIRE] and online at http://renormalization.com/19a3/.

[16] D. Anselmi, Fakeons, Microcausality And The Classical Limit Of Quantum Gravity, Class. Quant. Grav. 36 (2019) 065010 [arXiv: 1809.05037] [INSPIRE] and online at http://renormalization.com/18a4/.

[17] C.G. 't Hooft and M.J.G. Veltman, Diagrammar, NATO Sci. Ser. B 4 (1974) 177 [CERN-73-09] [INSPIRE].

[18] N.N. Bogoliubov and D.V. Shirkov, Introduction to the theory of quantized fields, Interscience Publishers, New York U.S.A. (1959).

[19] H. Lehmann, K. Symanzik and W. Zimmermann, On the formulation of quantized field theories. II, Nuovo Cim. 6 (1957) 319 [INSPIRE].

[20] C.G. Bollini and J.J. Giambiagi, Lowest order divergent graphs in $\nu$-dimensional space, Phys. Lett. B 40 (1972) 566 [InSPIRE].

[21] G. 't Hooft and M.J.G. Veltman, Regularization and Renormalization of Gauge Fields, Nucl. Phys. B 44 (1972) 189 [inSPIRE].

[22] G.M. Cicuta and E. Montaldi, Analytic renormalization via continuous space dimension, Lett. Nuovo Cim. 4 (1972) 329 [InSPIRE].

[23] R.E. Cutkosky, Singularities and discontinuities of Feynman amplitudes, J. Math. Phys. 1 (1960) 429 [INSPIRE].

[24] M.J.G. Veltman, Unitarity and causality in a renormalizable field theory with unstable particles, Physica 29 (1963) 186 [INSPIRE].

[25] L.D. Landau, On analytic properties of vertex parts in quantum field theory, Nucl. Phys. B 13 (1959) 181 [Sov. Phys. JETP 10 (1959) 45] [Zh. Eksp. Teor. Fiz. 37 (1959) 62] [InSPIRE].

[26] W.L. van Neerven, Dimensional Regularization of Mass and Infrared Singularities in Two Loop On-shell Vertex Functions, Nucl. Phys. B 268 (1986) 453 [InSPIRE].

[27] M. Böhm, H. Spiesberger and W. Hollik, On the One Loop Renormalization of the Electroweak Standard Model and Its Application to Leptonic Processes, Fortsch. Phys. 34 (1986) 687 [INSPIRE].

[28] M. Consoli and W. Hollik, Electroweak radiative corrections for $Z$ physics, in $Z$ physics at LEP 1. Volume 1: Standard physics, G. Altarelli, R. Kleiss and C. Verzegnassi eds., CERN, Geneva Switzerland (1989) [CERN-89-08-V-1].

[29] D. Anselmi and M. Piva, A new formulation of Lee-Wick quantum field theory, JHEP 06 (2017) 066 [arXiv:1703.04584] [INSPIRE] and online at http://renormalization.com/17a1/.

[30] S.W. Hawking and T. Hertog, Living with ghosts, Phys. Rev. D 65 (2002) 103515 [hep-th/0107088] [INSPIRE]. 
[31] D. Anselmi and M. Piva, The Ultraviolet Behavior of Quantum Gravity, JHEP 05 (2018) 027 [arXiv: 1803.07777] [INSPIRE] and online at http://renormalization.com/18a2/.

[32] D. Anselmi and M. Piva, Quantum Gravity, Fakeons And Microcausality, JHEP 11 (2018) 021 [arXiv: 1806.03605] [INSPIRE] and online at http://renormalization.com/18a3/.

[33] S. Weinberg, Ultraviolet divergences in quantum theories of gravitation, in An Einstein centenary survey, S. Hawking and W. Israel eds., Cambridge University Press, Cambridge U.K. (1979).

[34] I.G. Avramidi and A.O. Barvinsky, Asymptotic freedom in higher derivative quantum gravity, Phys. Lett. B 159 (1985) 269 [inSPIRE].

[35] N. Ohta, R. Percacci and A.D. Pereira, Gauges and functional measures in quantum gravity II: Higher derivative gravity, Eur. Phys. J. C 77 (2017) 611 [arXiv:1610.07991] [InSPIRE].

[36] A. Salvio and A. Strumia, Agravity up to infinite energy, Eur. Phys. J. C 78 (2018) 124 [arXiv: 1705.03896] [INSPIRE]. 